\title{
Design of Wireless Networked Electricity Pole Line Multi-Fault Monitoring System
}

\author{
P.Priya, R.Karuppasamy, R.RagavaRaja, B.Pradeep
}

\begin{abstract}
Over the year's Electrical shock on EB pole has become Defenseless to a transmission system. The main revelation of this project is to Defense from the electric shock. This plan is aimed at measuring the current flow in the transmi ssion line at the pole point and tracking parameters such as volt age sensors, current sensors in street lamps with pole position s ensors. The current and voltage sensors are continuously read the real-time values and send the analog values to the microcontroller present in the kit, if any one of the parameters levels goes beyond its normal value like wire disconnection, lamp failure or pole slanting and also the supply at wire disconnected point will be terminated by relays. Values are uploaded to the IoT cloud by means of the communication module. From the IoT cloud, the values can be monitored in the substation. From this, we can save lives and protect them from electricity.
\end{abstract}

Keywords: Sensor, Microcontroller, IoT cloud, Communication Module, Relay.

\section{INTRODUCTION}

Electricity is a set of phenomena associated with the presence and motion of matter that has the property of electric charges such as lightning static electricity, electric discharge and etc. The electricity transmission has developed a long way from the point where it started, when Benjamin Franklin discovered it in May 1752, but still there exist several problems in the transmission of the electricity to domestic consumers. There are many faults in transmission line disconnection or cut off due to external forces, voltage sag, under voltage, over voltage surge, unbalanced phases, reversed power, slanting pole, open circuit faults, short circuit faults, etc. These faults will normally affect the transmission efficiency but could also lead to loss of lives if some of the faults left unnoticed. There are many methods that are developed for the safety of consumers from the faults like surges and transmission line cut off. And still, there is no efficient way to implement those methods. This monitoring system is designed to protect the consumers from the faults that could possibly happen. Since

Revised Manuscript Received on December 05, 2019.

* Correspondence Author

Mrs.P.Priya*, Department of Electrical and Electronics Engineering, Kalasalingam Academy of Research and Education, Anand Nagar, Krishnankoil, Virudhunagar, India. Email: priya.p@klu.ac.in

Mr.R.Karuppasamy, Department of Electrical and Electronics Engineering, Kalasalingam Academy of Research and Education, Anand Nagar, Krishnankoil, Virudhunagar, India. Email: sweetyrksamy@ gmail.com

Mr.R.RagavaRaja, Department of Electrical and Electronics Engineering, Kalasalingam Academy of Research and Education, Anand Nagar, Krishnankoil, Virudhunagar, India. Email: ragavaramar@gmail.com

Mr.B.Pradeep, Department of Electrical and Electronics Engineering, Kalasalingam Academy of Research and Education, Anand Nagar, Krishnankoil, Virudhunagar, India. Email: b.n.b.pradeep@gmail.com the development of wireless network everything is getting upgraded and so does the electric grid system. But the transmission line monitoring has yet to be monitored using the wireless network in our region. The wireless monitoring could be monitored easily through IoT. The IoT is a popular platform to monitor and control things in a handy way.

There many features in IoT like monitoring real- time values, sending alert messages and even load control. The possibilities of wireless systems and IoT could be very helpful in transmission system monitoring. The microcontroller advancement could also be a key for a proper monitoring system.

The usual cause of power cut in remote areas is due to carelessness and laziness of people who maintain the transmission system in those areas. This might not seem like a bigger problem but it is dangerous to the people who live around the area. In this study, a newly conceived fault detection system is designed, built and defined for electrical pole components. The aim of designing and developing a fault detection system is to provide an immediate and effective solution when any part of the electrical of a utility pole component is detected faulty.

To solve the problem, the proposed system is integrated with the fault finding system network communication system and global mobile service communication to create a complete architecture technique.

In the proposed design, specifically, the circuit fault sensing which diverges a HIGH signal from any failed device. Still fault detection circuit retain the HIGH signal from the failed systems, also to detect which system has the fault that explores the information and process from the fault sensing circuit. The proposed architecture a novel established system assimilating a keen microcontroller that can execute different deviation signals from the fault sensing circuitry to instantaneously rectify the fault and reducing the disruption time for preservation. Fault detection circuit also maintains the HIGH signal from failed systems to determine which device has the fault which examines the details and processes from the fault sensing circuit..The proposed architecture is a new system that assimilates a detailed micro controlling.

\section{LITERATURE REVIEW}

In the literature, few simulation models are manipulated. Benazir Fateh et al., the Wireless Sensor Network (WSN) has established a system for real-time situational awareness of the electrical transmission network. When WSNs are able to monitor wide geographical locations in a cost-effective manner, there are considerable technical problems. 
In the literature, few simulation models are manipulated. Benazir Fateh et al., the Wireless Sensor Network (WSN) has established a system for real-time situational awareness of the electrical transmission network. When WSNs are able to monitor wide geographical locations in a cost-effective manner, there are considerable technical problems. The low-power, low-data devices impact bandwidth and latency gaps. The main objective in this work is to develop a wireless network that delivers physical measurements in real-time for the optimal preventive or corrective control action. For network design, an optimization process is formulated in order to reduce deployment and operating costs when the end-to-end latency and bandwidth constraints of the data flow are achieved. For the designing of network, they are framed an optimization task with the aim of reducing the installation and operational costs when completed the end-to-end latency and bandwidth constraints of the data flow. They went through a hybrid hierarchical network architecture based on a mix of wired, cellular and wireless technologies that could ensure low-cost real-time monitoring of data. To discover the optimal location of cellular assisted transmission towers, they are developed a placement problem. Therefore, the latest test outcomes of the optimization solution for the different circumstances. Our concept is universal and describes real-world situations of asymmetric data generation of sensors, untrusted behavior of wireless networks, non-uniform cellular network coverage, etc. The research shows that a monitoring framework for transmission lines using WSN is certainly possible using available technologies. The results indicate that wireless link bandwidth can be a preventive factor to reduce costs.

"V.Thiyagarajan et al., Recommends an innovative design to develop an AVR microcontroller-based system to track the current, temperature and voltage of a transmission transformer in a substation and to maintain the device against an increase in the specified parameters. Providing protection for the distribution transformer can be adept with the help of RF Communication by shutting down the entire unit. In addition, the device displays the similar on a PC located at the main station in the remote area. It also has the ability to detect the breakdowns caused by overvoltage, high temperature and overloadThis architecture usually consists of two units, one in the substation unit, referred to as a transmitter and display unit, and the other as a command unit is the main stationThe transmitter and display systems in the substation are where AVR microcontroller continuously controls temperature voltage and current and is shown using the display unit. The control unit at the main station that denotes the PC and an RF receiver which absorbs the RF signals sent by the transmitter and responds to the signal received. The proposed architecture has generally made it user-friendly to locate the transmission transformer that is affected by any short or open circuit and to raise temperatures. The main objective is to constantly track the electrical parameters and thus prevent the burning of the transmission transformer or power transformer due to over-temperature, high voltage input and overload. If any of these values increases beyond the limit then the entire unit is shut down by the designed controlling unit. KomiAgbesi et al., Research focused on smart GSM-based fault detection and location system was used to recognize and locate the exact position where the fault occurred appropriately and accurately. It will allow a faster response time for technicians to repair the faults and will therefore be very useful in shielding transformers from accidents and damage. The system consists of a voltage transformer, a current transformer, a RS-232 connector and a GSM modem. This system automatically detects, analyzes and classifies these faults and then uses an impedance based algorithm technique to calculate the fault position from the control room. Eventually, the fault data is sent to the control room. In addition, the time taken to find a fault is dramatically corrected as the system automatically and reliably provides precise information about the location of the fault.

\section{PROPOSED SYSTEM}

In the transmission of electricity the common problem faced in remote areas is basically two. One is finding the line which is at fault condition or disconnected or broken and the other is the lineman directly has to go and fix the fault physically.

To overcome these difficulties many systems have been proposed in the past that's mostly based on gsm and it'll inform the fault location via text messages. In this proposed system the operator in the electricity board can control the power in every single post. When a specific fault occurs between two posts the fault will be noticed to the operator in the electricity board control center where the operator could view the fault location and could also terminate the power supply to that specific post without affecting the power supply to other posts located before the fault post. Many security issues in the smart meters are found out by the researchers. The reliability issue can be divided into three classes; they are attacks on physical hardware and attacks on data and attacks on the network.

This way the board will know the specific location of the fault and since the power can be controlled from the board there will be no danger for people in case of broken power lines which was a major problem before as the lineman has to turn off the supply manually if the fault is informed by some locals. The other problem is slanting pole, it's common in remote areas where the power will be transmitted from A long way and no warning will be given if the pole is sla nted because of some natural cause or ground conditions. A MEMS sensor is used to measure the pole axis and if th e pole axis is slanted from the vertical position, the board will note it.

These faults are reduced in our proposed system and the sa fest and most efficient method of monitoring and controlli $\mathrm{ng}$ them is provided by IoT.

\section{METHODOLOGY}

The voltage and current sensing circuits will be connected to each pole which will measure the voltage and current passing through the pole. 
The MEMS sensor is also connected to the pole to measure the position of the pole. These sensors values are gathered in an interfacing unit and from there it is sent to Arduino UNO. The microcontroller which is used is Arduino UNO which more compatible for usage. It has more analog pins and digital pins so we can able to connect a lot of analog sensors to get the data. The values which are obtained from the sensors and calculated values are continuously shown upon the LCD display which is connected with the microcontroller. In addition to that, the values will be transferred to Node MCU through Receiving (RX) and Transmitting (TX) pins and the Node MCU will send the data to Cloud MQTT which is an IoT platform. The current sensing circuit is used to detect the lamp failure in the street lamp connected to the pole. The voltage sensor is used to detect line disconnection on breakage.

We can also detect any sudden drop or change in the transmission since it's constantly monitored through IoT. Whenever any attacks happened in the IoT cloud or smart meters the algorithm which is present in the substation will indicate so that the substation controller can identify the attacks thereby tripping of the concern smart meter consumer is penalized.

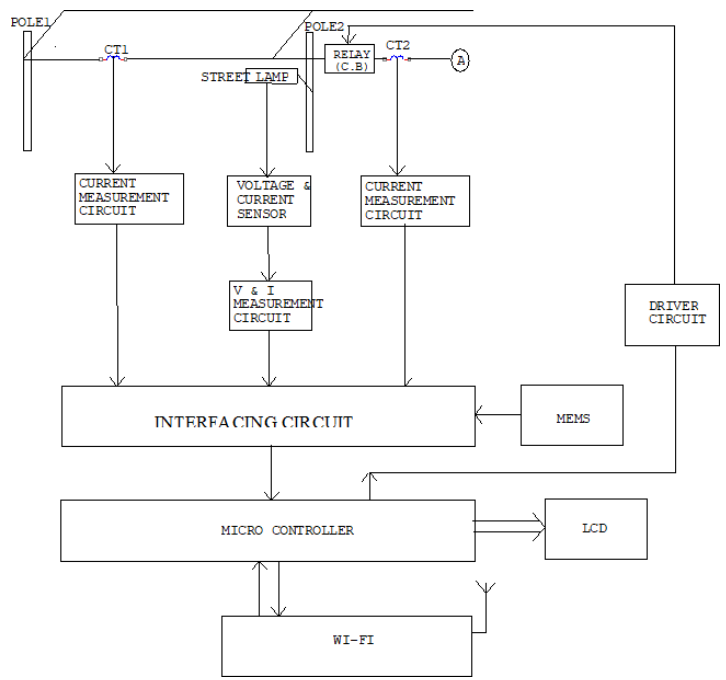

Fig. 1. Block Diagram of Multi-fault Monitoring System

\section{RESULT AND DISCUSSION}

The entire hardware connection is shown in Fig (2),

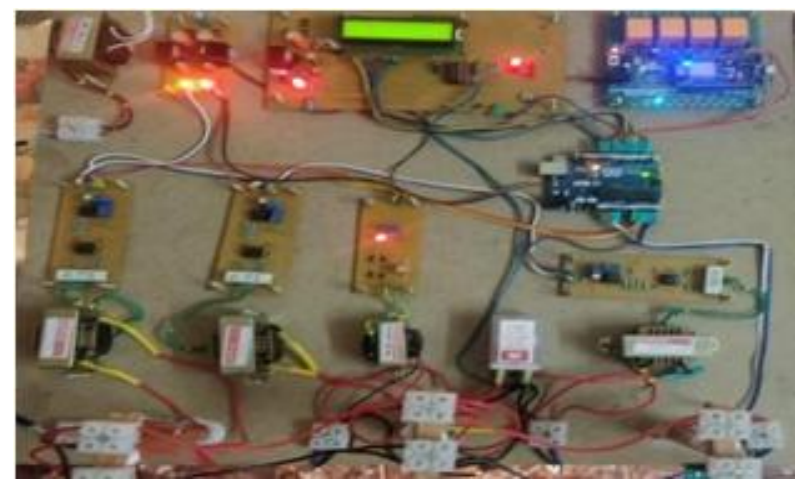

Fig. 2. Hardware connection of the Multi-fault Monitoring System
Both microcontrollers and the Wi-Fi communication module are connected with the computer so that the coding can be uploading to the respective controllers. The LCD is connected to Arduino boards with the digital pins. Each current and voltage sensing circuit is connected to analog pins A0 to A5. In figure 3 values are shown in LCD display.

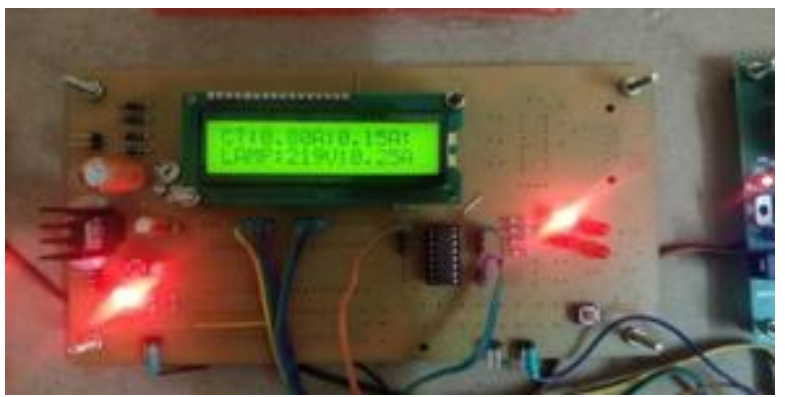

Fig. 3. LCD Display

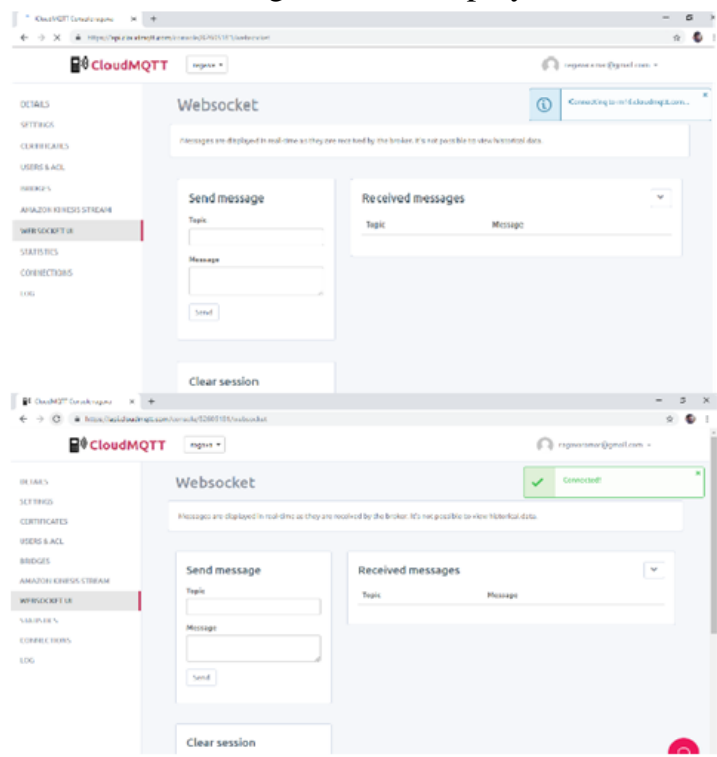

Fig. 4. Multi-fault Monitoring System is shown in MQTTCloud

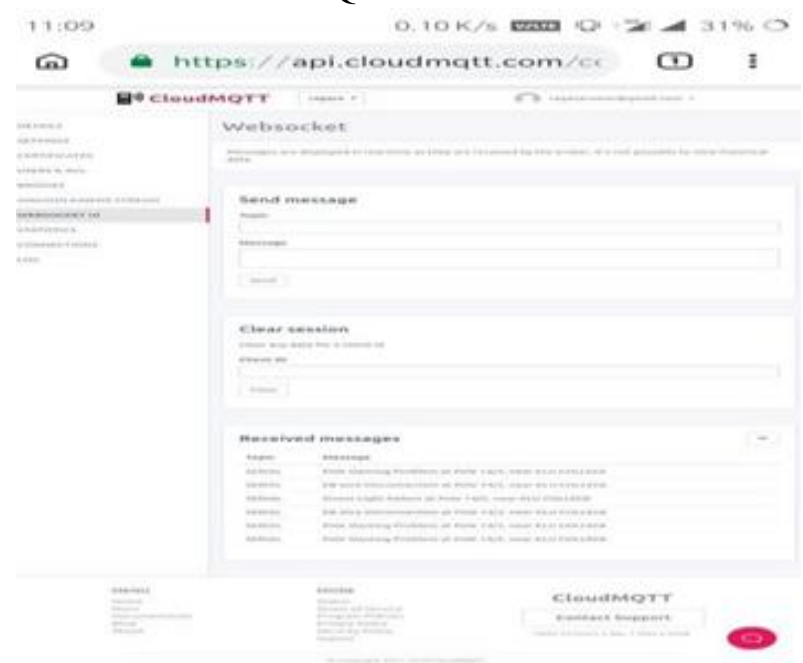

Fig. 5. Multi-fault Monitoring System final output

The LCD display shows the current and voltage value in real time. It also indicates the power consumed by the lamp connected in the specific pole. 
The cloud MQTT acts as a platform to communicate with the electricity board. The values displayed in the LCD are also sent to node MCU. The node MCU will upload the value to the cloud MQTT. The values can be monitors real time and if any fault occurs in the system like lamp fault or slanting pole it'll be notified immediately in the cloud MQTT with the location of the pole.

\section{CONCLUSION}

In many rural areas, there are still power cut problems due to broken transmission lines and lives are lost due to the laziness of the officials who fail to find the location of the fault. By this proposed system they don't need to search for fault in total area and can concentrate on a single pole to pole connection in the notified area. It'll be really helpful to update the rural power transmission system.

\section{REFERENCES}

1. Benazir Fateh\&ManimaranGovindarasu, "Wireless Network Design ForTransmission Line Monitoring In Smart Grid", Ieee Transaction On Smart Grid, Volume 4, No.2,2013.

2. IuHua, Zhang Junguo, Lin Fantao“ Internet Of Things Technology And Its Applications In Smart Grid"e vol12,No2,2014.

3. Zheng, T.; Siebers, P.-O.; Aickelin, U. emulating electricity usage industrial buildings: An agent-based approach. Energy Build. 2011, 43, 2882-2892.

4. Arduino connection with current sensor available online link:HTTP:// henrysbench .capnfatz.com/ henrys-bench /Arduino-currentmeasurements/the-acs712-current-sensor-with-an-Arduino/

5. Devidas, A.R. Ramesh, M.V., "Wireless Smart Grid Design ForMonitoring And Optimizing Electric Transmission In India”, Fourth International Conference On Sensor Technologies And Applications (Sensorcomm), Ieee 2010.

6. ZhaiZi-Nan And Gui Wei-Feng, "Research On Monitoring Power System Faults By Wireless SensorNetwork", Research On Monitoring Power System Faults By Wireless Sensor Network,2010

7. Sending data from Arduino to IoT cloud, Available link:https://github.com/ MathWorks/ thingspeak-Arduino

8. Arduino and nodemcu connection for communication, Available link:HTTP: //henrysbench. capnfatz. com/henrys-bench /Arduinoprojects-tips-and-more /connect- nodemcu- esp- 12e- to- wifi-routerusing- Arduino-ide/

\section{AUTHORS PROFILE}

Mrs.P.Priya is a Assistant Professor in Department of Electrical and Electronics Engineering in Kalasalingam Academy of Research and Education. Received M.E. in Power Electronics \& Industrial Drives from Sathyabama University in 2010. Then her B.E. in Electrical and Electronics Engineering from Sethu Institute of Technology in 2005. She has published 4 International journals and 1 Book Chapter.

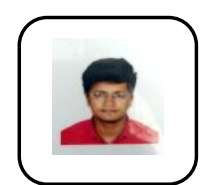

Mr.R.Karuppasamy is a Student in Department of Electrical and Electronics Engineering in Kalasalingam Academy of Research and Education. Studying B.E. in Electrical and Electronics Engineering at Kalasalingam Academy of Research and Education

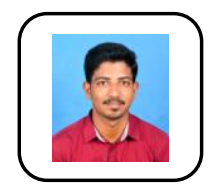

Mr.R.RagavaRaja is a Student in Department of Electrical and Electronics Engineering in Kalasalingam Academy of Research and Education. Studying B.E. in Electrical and Electronics Engineering at Kalasalingam Academy of Research and Education.

Mr.B.Pradeep is a Student in Department of Electrical and Electronics Engineering in Kalasalingam Academy of Research and Education. Studying B.E. in Electrical and Electronics Engineering at Kalasalingam Academy of Research and Education 